\title{
Dynamic pricing of network goods with boundedly rational consumers
}

\author{
Roy Radner ${ }^{a, b, 1}$, Ami Radunskayac, and Arun Sundararajan ${ }^{\text {b,d }}$ \\ ${ }^{a}$ Economics Department, ${ }^{b}$ Information, Operations, and Management Sciences Department, Stern School, and ${ }^{\mathrm{d}}$ Center for Urban Science and Progress, \\ New York University, New York, NY 10012; and 'Mathematics Department, Pomona College, Claremont, CA
}

Contributed by Roy Radner, October 30, 2013 (sent for review July 21, 2013)

We present a model of dynamic monopoly pricing for a good that displays network effects. In contrast with the standard notion of a rational-expectations equilibrium, we model consumers as boundedly rational and unable either to pay immediate attention to each price change or to make accurate forecasts of the adoption of the network good. Our analysis shows that the seller's optimal price trajectory has the following structure: The price is low when the user base is below a target level, is high when the user base is above the target, and is set to keep the user base stationary once the target level has been attained. We show that this pricing policy is robust to a number of extensions, which include the product's user base evolving over time and consumers basing their choices on a mixture of a myopic and a "stubborn" expectation of adoption. Our results differ significantly from those that would be predicted by a model based on rational-expectations equilibrium and are more consistent with the pricing of network goods observed in practice.

behavioral economics | increasing returns | diffusion | optimal control | industrial organization

A good or service is said to display network effects, or more briefly is called a network good, if the value to each consumer of the good or service is influenced by the consumption choices made by some or all other consumers. Economic models of network goods typically assume the following "unboundedly" rational consumer behavior: Consumers immediately react to each observable strategic decision made by a seller, by forming a common expectation of demand and making their consumption choices based on this expectation, which is then realized in equilibrium. This solution is commonly referred to as satisfying "fulfilled expectations" or as a rational expectations equilibrium. In the case of a monopoly, which is the context of the present paper, the seller uses the fulfilled expectations demand function to calculate her initial profit-maximizing price, which is then held constant in time.

Some notion of rationality is at the base of most current economic analysis. However, in the specific case of network goods, the requirement of rational expectations equilibrium imposes heavy demands on the cognitive abilities of consumers. Furthermore, the model typically makes predictions that are unrealistic.

In this paper we explore how the predictions of models of network effects change under assumptions about consumer rationality that seem more realistic. We do so by presenting a few alternative models of a monopoly market for a service with network effects, in which $(i)$ consumers do not (all) immediately react to every change in the seller's price and, furthermore, $(i i)$ make their consumption choices based on a "boundedly rational" assessment of expected demand. We use these models to study the resulting dynamic pricing problem for the monopolist. The adoption choices of the consumers continuously influence the rate at which demand adjusts over time, and the monopolist therefore chooses a trajectory of prices that maximizes her discounted stream of profits.

In our benchmark model, consumers are myopic in their expectations, and we show that the monopolist's optimal pricing trajectory is generated by a target policy with the following properties: When current demand is below the target, the price is low; when current demand is above the target, the price is high; and when current demand is at the target, the price is chosen to keep demand stationary. The target could be interpreted as the level of adoption below which the monopolist invests in building a user base and above which the monopolist profits from exploiting her installed base. The result thus prescribes a form of penetration pricing that is not uncommon in markets for network services. We also show that the optimal demand target with myopic consumers is lower than the level of demand predicted by the corresponding rational expectations equilibrium.

It is beyond the scope of this paper to survey the empirical literature on the pricing of network goods. Furthermore, our theoretical model is too simple to cover the complexities that one usually encounters in such markets. For one thing, pure monopolies are rare, although the occurrence of firms with considerable market power is not. For another, markets for network goods typically include several categories of customers and take the form of "twosided markets," etc. We confine ourselves here to describing two historical examples that, although having some departures from pure monopoly, are close enough to our model to give qualitative support to our general conclusions about pricing strategy.

An early example of a network good was telephone service, both local and long distance (LD). Based on Alexander Graham Bell's 1876 patent of the telephone, AT\&T (The Bell System) was a legal monopolist until the patent's expiration in 1894. AT\&T remained a monopolist in LD through acquisition of additional patents, as well as in local service in many areas. Thus, during the early years of the 20th century, AT\&T was the dominant firm in local service in many areas and the dominant firm in LD in the country. In this period, it gradually increased its dominance, attaining the status of an unregulated monopoly in many local jurisdictions (1). This period ended in 1913 when AT\&T settled an antitrust suit by the US Attorney General with an out-ofcourt agreement (the "Kingsbury Commitment"), at which time its business began to be partially regulated (ref. 2, p. 10).

With respect to our model, the case of AT\&T up to 1894 fits fairly well, and after that until 1913 AT\&T had considerable monopoly power. Customers were classified as "residence" or "business," with separate prices for each category. According to

\section{Significance}

A consumer's demand for a network good depends on the demands of other consumers, and therefore choosing this demand optimally poses a cognitive challenge for most consumers. In our model of pricing a network good, consumers display "bounded rationality" (in Herbert Simon's sense), and the vendor chooses a dynamic price path to maximize the present value of profit. The result is a price and quantity path that differs significantly from that predicted by standard economic theory and is closer to empirical observations.

Author contributions: R.R., A.R., and A.S. designed research, performed research, analyzed data, and wrote the paper.

The authors declare no conflict of interest.

Freely available online through the PNAS open access option.

${ }^{1}$ To whom correspondence should be addressed. E-mail: rradner@stern.nyu.edu.

This article contains supporting information online at www.pnas.org/lookup/suppl/doi:10. 1073/pnas.1319543110/-/DCSupplemental. 
Gabel, “. . . at the start of the century AT\&T's managers believed that residential service should be provided at a rate that was less than the cost of direct service. This 'loss' was more than made up by the higher charges that could then be set for business lines; . . the intent was to bring new customers onto the network and thereby raise the value to the existing customers" (ref. 1, p. 545). Note the reference to the "network goods effect" in the last sentence above. (However, it should be mentioned that there was some dispute among economists about whether this pricing policy was "predatory," i.e., intended to drive competitors out of business, an illegal practice, and Gabel presents evidence on this and possibly other anticompetitive behavior.)

A more recent example is PayPal. However, this is not a pure example either, because there are two classes of customers, individuals and businesses, and there is competition in the relevant market. PayPal provides a service that allows individuals to send money to other individuals and businesses via the Internet more cheaply and more conveniently than by check, bank transfer, or the usual credit card transactions. Initially, PayPal offered a small rebate to individuals who signed up for their service and charged nothing for payments made to other account holders. Also, the charges paid by businesses receiving money were significantly less than those charged by the standard credit card companies. This strategy was followed for a few years, during which their customer base grew rapidly. At this point PayPal started to make money by eliminating the rebates to new individual customers, narrowing the definition of "individual customer," and increasing the charges to business customers, although the latter were still less than standard credit card charges, and "individual" customers were not charged for sending money. By the time it entered the profitable phase, PayPal had established a dominant position in its market. (For details, see ref. 3.)

In our theoretical models, we assume that a consumer's instantaneous utililty (surplus) of subscribing to the service is a linear function, increasing in the total nunber of current subscribers and decreasing in the current price. The marginal utility of the total number of subscribers, denoted by $\theta$, is distributed in the population of potential consumers, with a cumulative distribution function (cdf) that is convex or concave. The extension of the analysis to a more general class of cdfs remains a project for future research. However, we do show that, for a fairly general class of cdfs, the optimal policy for the rational expectations model cannot be the steady state of our model with myopic consumers.

Our subsequent results extend the analysis in two directions, for the special case of the uniform cdf of $\theta$. First, we examine how the monopolist's optimal price trajectory varies when the population of consumers evolves over time. That is, in each period, a constant fraction of consumers is (exogenously) replaced by new ones, at a rate determined by a parameter $c$ that is proportional to the fraction of consumers replaced in each period. The monopolist's optimal pricing trajectory continues to be generated by a target policy with the same qualitative properties as the one in the "benchmark myopic case," although with a strictly lower optimal demand target. Moreover, in this model, the price that keeps demand stationary at any desired level is progressively lower as $c$ increases.

In a second extension we examine how the monopolist's optimal price trajectory varies when the expectation of demand formed by each consumer (who pays attention) is a weighted average of the myopic expectation and an exogenously specified "stubborn" expectation. Our final theorem establishes that, again, the monopolist's optimal pricing trajectory continues to be generated by a target policy with the same qualitative properties as the one in the basic myopic case, but again with a lower target demand level. The target increases as consumers become less stubborn, eventually converging to the target demand level of the policy for purely myopic consumers.

To sum up the preceding discussion, our model is too simple to fit all of the aspects of the historical examples, but its results provide a qualitative insight into pricing behavior that goes beyond that provided by standard economic theory using "unboundedly rational" consumers.

We have organized the rest of this paper as follows. The next section describes our model of bounded rationality, our underlying discrete-time model, and the derivation of its continuous-time counterpart. The following section analyzes our "base" model with myopic consumers. In it we describe the optimal price trajectory for this model and contrast it with that predicted by the rational expectations equilibrium. We then extend the base model in the two ways described above. We conclude with a summary and comments on our results and sketch a program of future research. The final section contains bibliographic remarks and the list of references. Proofs of the theorems, and further bibliographic remarks, can be found in SI Text.

\section{Overview of the Model}

We introduce our continuous-time formulation of a monopolist's market for a network good as the limiting case of a discrete-time model. We emphasize that our discussion of the discrete-time model and the passage to the limit is designed only to motivate the continuous-time formulation and is therefore "heuristic" in the sense that we leave out many of the mathematical details that would be required for a more rigorous discussion (see comments below).

In the discrete-time model, the length of each period is $h$, and time is divided into periods, $n=0,1,2, \ldots$ ad inf. Calendar time is denoted by $t$, a nonnegative real number, so that period $n$ begins at $t=n h$ and ends at $t=(n+1) h$. A network good is provided by a monopolist, who sells the good in units of one period. (Think of the good as a service.) At the beginning of period $n$, the monopolist announces a price $p(t)$ (per unit time) for the time interval $n h \leq t<(n+1) h$. We assume that the price is constrained to be nonnegative and is bounded above (more on this later). A continuum of consumers is indexed by a "type" parameter $\theta$. We take the mass of the consumers to be unity and the set of types to be the unit interval. If a consumer of type $\theta$ buys the service for one period, and the total mass of consumers who buy the service in that period is $q(t)$, then the consumer's (incremental) instantaneous utility during that period will be equal to

$$
\theta q(t)-p(t)
$$

Note that $p(t)$ and the decision of each consumer are constant during any one period. Let $F$ be the cumulative distribution function of $\theta$; i.e., the fraction of consumers with type less than or equal to $\theta$ is $F(\theta)$. For simplicity, we assume $F$ to be absolutely continuous and strictly increasing on the unit interval. [Note that the mathematical problems of dealing with a continuum of random variables are well known, and (as noted above) the preceding story is only a heuristic description of a model. See ref. 4 for more details.]

The first aspect of our model of bounded rationality specifies how consumers form their expectation of demand for the coming period. Specifically, we assume that each consumer who observes the price $p(t)$ at the beginning of period $n$ makes the same prediction, $q_{E}(t, h)$, of the total demand in period $n$. Therefore, a consumer of type $\theta$ who observes $p(t)$ will buy the good if and only if $\theta q_{E}(t, h) \geq p(t)$. In this paper we discuss several models of expectation formation.

The second aspect of our model of bounded rationality is the rate at which consumers react to price changes. In the typical discrete-time model, there is an implicit delay in the decision of each participant to the receipt of information about the decisions of other players, namely the length of the time interval in the model. This delay is important in the dynamics of our model. We adopt a specific model of this delay, which leads to a "smooth" model of dynamics in the limiting case of continuous time. 
At the beginning of each period (with two exceptions noted below), a "random" fraction $k h$ of consumers of each type "pay attention to" the current price $p(t)$. Correspondingly, the remaining fraction $(1-k h)$ of consumers of each type do not pay attention to the monopolist's price announcement, and their choice remains unchanged in period $n$ from that in period $(n-1)$. Note that an equal fraction $k h$ of consumers of each type "pay attention" in each period and that the magnitude of this fraction depends on the length of the interval $h$. One might therefore interpret $k$ as measuring a "rate of attention" of consumers to price changes or a "rate of adjustment." Thus, the average time between successive price checks by a consumer is $(1 / k)$.

The first exception to our model of bounded attention occurs when $p(t)>q_{E}(t, h)$. In this case, we assume that $q(t)=0$. The rationale is that if $p(t)>q_{E}(t, h)$ and every consumer expected the quantity demanded to be $q_{E}(t, h)$, then no consumer would want to buy the good in period $t$. In a sense, the occurrence of " $p(t)>q_{E}(t, h)$ " is a "wake-up call" for all consumers. Hence we assume directly that

$$
0 \leq p(t) \leq q_{E}(t, h)
$$

(Note that some upper bound on price is desirable to ensure that the model is mathematically well behaved. See ref. 5 for details.)

The second exception to our model of bounded attention occurs when $q_{E}(t, h)=0$. From the above constraint on $p(t)$, it would follow that $p(t)=0$. For technical reasons, we postpone dealing with this case until a later section.

To highlight how the dynamics of the system depend on the parameter $h$, we change the notation slightly and let $q_{h}(t)$ denote the actual total demand at time $t=n h$. We now derive a difference equation for $q_{h}(t)$ in the case in which both $q_{E}(t, h)$ and $q_{h}(t)$ exceed 0 . Let $w_{h}(\theta, t)$ denote the demand (per consumer) from consumers of type $\theta$ in period $t$. Thus

$$
q_{h}(t)=\int_{0}^{1} w_{h}(\theta, t) d F(\theta) .
$$

Recall that a "fraction" $k h$ of consumers of type $\theta$ pay attention to $p(t)$, form a shared expectation of demand $q_{E}(t, h)$, and decide whether or not to adopt the product for period $n$. Therefore, if $\theta \geq\left[p(t) / q_{E}(t, h)\right]$, each consumer in this fraction $k h$ adopts the product, and if $\theta<\left[p(t) / q_{E}(t, h)\right]$, then none of these consumers adopt the product. Because the remaining fraction $(1-k h)$ continue to do in period $n$ what they were doing in period $n-1$, it follows that

$$
w_{h}(\theta, t)=\left\{\begin{array}{cc}
k h+(1-k h) w_{h}(\theta, t-h), \quad \theta \geq p(t) / q_{E}(t, h), \\
(1-k h) w_{h}(\theta, t-h), \quad \theta<p(t) / q_{E}(t, h) .
\end{array}\right.
$$

The last two expressions imply

$$
\begin{aligned}
q_{h}(t) & =\int_{0}^{1}(1-k h) w_{h}(\theta, t-h) d F(\theta)+\int_{p(t) / q_{E}(t, h)}^{1} k h d F(\theta) \\
& =(1-k h) q_{h}(t-h)+k h\left(1-F\left[\frac{p(t)}{q_{E}(t, h)}\right]\right) .
\end{aligned}
$$

Our continuous-time model is obtained by letting the length $h$ of the interval in the discrete-time model tend to zero. Recall that $q_{E}(t, h)$ is the predicted demand in period $n$, where $n h \leq t<(n+1) h$, and define

$$
q_{E}(t)=\lim _{h \rightarrow 0} q_{E}(t, h) .
$$

Assume that $q_{E}(t)$ is well defined and depends at most on the current demand and price, $q(t)$ and $p(t)$, respectively. A straightforward calculation yields the differential equation for the demand trajectory.

Lemma 1. If at time $t>0$ the demand and price are $q(t)$ and $p(t)$, respectively, and

$$
0<q(t)<1, \quad 0 \leq p(t) \leq q_{E}(t)<1,
$$

then the time rate of change of demand is specified by

$$
q^{\prime}(t)=k\left\{1-F\left[\frac{p(t)}{q(t)}\right]-q(t)\right\} .
$$

Completing the specification of the law of motion at the boundary values is postponed to the following sections, where different specifications of the prediction function, $q_{E}$, are formulated. Also, it is assumed that the initial values, $q(0)$ and $q_{E}(0)$, are given.

The monopolist wants to choose a price trajectory $p(t)$ to maximize her profit. Assume, for simplicity, that her (marginal) cost of providing the service is zero (see below); then her total discounted (variable) profit is

$$
\int_{0}^{\infty} e^{-r t} p(t) q(t) d t
$$

where $r>0$ is her given discount rate, and $q(t)$ evolves according to the model.

The precise formulation of the maximization problem will depend on the particular cases we consider in the following sections.

\section{The Model with Myopic Consumers}

We now describe the monopolist's optimal price trajectory for a class of models of bounded rationality in which consumers are myopic; i.e., their expected demand in the "immediate future" is equal to the current demand; i.e., $q_{E}(t)=q(t)$.

It is convenient to take the monopolist's control variable, $\boldsymbol{a}(t)$, to be the ratio of price to expected quantity demanded, and hence

$$
\boldsymbol{a}(t)=\frac{p(t)}{q(t)}
$$

with the assumption that $0 \leq \boldsymbol{a}(t) \leq 1$.

For $0<q(t) \leq 1$, the law of motion of the quantity demand given by Lemma 1 is

$$
q^{\prime}(t)=k[1-F[\boldsymbol{a}(t)]-q(t)] .
$$

If $q(t)=0$, then the constraint $0 \leq p(t) \leq q(t)$ implies that $p(t)=0$, so that $\boldsymbol{a}(t)=0 / 0$. We therefore modify the law of motion in Lemma 1 as follows: With a slight abuse of notation, define

$$
m(q, a)=k[1-F(a)-q], \quad 0 \leq q \leq 1, \quad 0 \leq a \leq 1,
$$

and assume that the law of motion is

$$
q^{\prime}(t)=m[q(t), \boldsymbol{a}(t)], \quad 0 \leq q(t) \leq 1, \quad 0 \leq \boldsymbol{a}(t) \leq 1 .
$$

Note that the function $m$ is continuous everywhere in the unit square. In particular, $m(0,1)=0$, whereas $m(0, a)>0$ for $a<1$. Thus, this modification of the law of motion removes the discontinuity in the version contained in Lemma 1.

Given the quantity $q(t)$ and the control $\boldsymbol{a}(t)$, the monopolist's profit at time $t$ is $\boldsymbol{a}(t) q(t)^{2}$. Hence the monopolist's maximization problem is 


$$
\max _{\boldsymbol{a}} \int_{0}^{\infty} e^{-r t} \boldsymbol{a}(t) q(t)^{2} d t
$$

subject to the law of motion, Eq. 10 (with its constraints), and given the initial quantity, $q(0)$.

In the following subsection, we give a precise definition of a rational expectations equilibrium (REE) and show that, under suitable regularity conditions on the cumulative distribution function $F$, a REE cannot be a steady state of the system with myopic consumers and a profit-maximizing monopolist.

Rational Expectations Equilibrium Is Not Optimal. An alternative theory of consumer behavior is embodied in the concept of "rational expectations equilibrium." Imagine that, when faced with a price $p$, each consumer correctly predicts the total demand at that price and decides whether or not to subscribe on the basis of that prediction. Thus, the total demand at that price must satisfy

$$
q=1-F\left(\frac{p}{q}\right)=1-F(a)
$$

provided $q>0$. Suppose now that the monopolist chooses the price, say $p^{* *}$, to maximize $p q$ subject to the last equation as a constraint; call the corresponding demand, $q^{* *}$. Following standard terminology, we call such a pair $\left(p^{* *}, q^{* *}\right)$ a REE. Theorem 1 states that, for a sufficiently "regular" cdf $F$, a REE cannot be a steady state for an optimal trajectory with boundedly rational myopic consumers.

Recall that $F$ is absolutely continuous and strictly increasing, with $F(0)=0$, and $F(1)=1$. Hence Eq. 12 implicitly defines an absolutely continuous, strictly decreasing mapping, say $A$, from $q$ to $a$,

$$
A(q)=F^{-1}(1-q)
$$

The corresponding profit is

$$
v(q)=A(q) q^{2}
$$

Define $q^{* *}=\arg \max _{q} v(q)$ and $a^{* *}=A\left(q^{* *}\right)$.

Because $v(0)=0$, it follows that $q^{* *}>0$ and $0<a^{* *}<1$. For the purpose of this subsection, we say that $F$ is regular if $q^{* *}$ satisfies the usual first-order condition for a maximum of $v$,

$$
v^{\prime}\left(q^{* *}\right)=0
$$

Theorem 1. If $F$ is regular, then the optimal REE cannot be a steady state of an optimal dynamic price trajectory with boundedly rational myopic consumers.

In fact, in the cases discussed in this paper, the REE demand is larger, and the REE price is smaller, than the respective demand and price in a steady state of an optimal trajectory. In particular, we consider three cases for the distribution function: $F$ is strictly convex, $F$ is strictly concave, and the boundary case where $F$ is uniform.

Convex Distributions of Consumer Types. We first consider the case where the cumulative distribution function of consumer types is strictly convex. By an extension of Pontryagin's maximum principle, along the lines of ref. 6 , we can prove the following:

Theorem 2. If the cdf $F$ is strictly convex, then there is a unique optimal stationary policy, $\alpha(q)$, that is the solution of the differential equation

$$
a^{\prime}=\frac{F^{\prime}(q)}{q F^{\prime \prime}(a)}\left[2 k\left(1-F(a)-q+a F^{\prime}(a)\right)-(r+k) q\right] .
$$

Furthermore, $\alpha(q)$ is increasing as a function of $q$ and, under this optimal policy, $q(t)$ converges monotonically in finite time to a target demand, $q^{T}$, with target policy $\alpha^{T}$. This target is given by

$$
q^{T}=1-F\left(a^{T}\right)=\frac{2 k}{r+3 k}\left[1-F\left(a^{T}\right)+a^{T} F^{\prime}\left(a^{T}\right)\right]
$$

where

$$
a^{T}=\frac{r+k}{2 k F^{\prime}\left(a^{T}\right)} .
$$

We also show that the target demand is strictly less than the REE if $F$ is strictly convex and regular. Fig. 1 illustrates this result in the case $F(a)=a^{2}$.

Theorem 3. If the cdf $F$ is regular and strictly convex, then the target demand, given by Eq. 17, is less than or equal to the REE demand, given by Eq. 14, with equality in the limit as $r \rightarrow 0$.

Uniformly Distributed Consumer Types. In the special case in which $\theta$ is distributed uniformly on the unit interval the optimal policy has the following special form, which we call a jump target policy. For a given number, say $s$, with $0<s \leq 1$, the jump target policy with target $s$ is defined by

$$
\pi(q)= \begin{cases}0, & q<s \\ 1-s, & q=s \\ 1, & q>s\end{cases}
$$

For example, if the initial demand is less than the target, the demand will increase, and the revenue will remain zero, until the demand reaches the target, after which the revenue will remain at $(1-q) q^{2}$. Note that there is a trade-off between reaching a higher target and getting there sooner. Several demand trajectories are shown in SI Text.

Theorem 4. The optimal target policy $\pi^{*}$ is optimal among all policies, and the optimal target is

$$
\sigma \equiv \frac{2 k}{3 k+r}
$$

The proof uses the Bellman-Blackwell method of dynamic programming. A by-product of the method is that the value function, which describes the monopolist's maximum discounted profit as a function of the initial state $q$, is given by the formula

$$
V_{\pi^{*}}(q)=\frac{1}{r}\left[(1-q)^{-(r / k)}\right]\left[\sigma^{2}(1-\sigma)^{\left(1+\frac{r}{k}\right)}\right],
$$

and $V_{\pi^{*}}(q)$ is increasing in $k$ if the initial demand $q$ is less than the optimal target, $\sigma$.

It is straightforward to verify that the demand in the REE for the uniform case is $2 / 3$, which is larger than the target in the optimal policy.

Concave Distributions of Consumer Types. In the case where the cdf, $F$, is strictly concave, there is no optimal stationary policy in the classical sense. However, we can show that there is an optimal "measure-valued policy" that is independent of $F$ and whose structure is very similar to that of the optimal target policy in the uniform case.

Let $F$ be an absolutely continuous cdf that is strictly increasing and strictly concave on the unit interval, with $F(0)=0$ and $F(1)=1$. We extend the set of permissible controls to include those that 


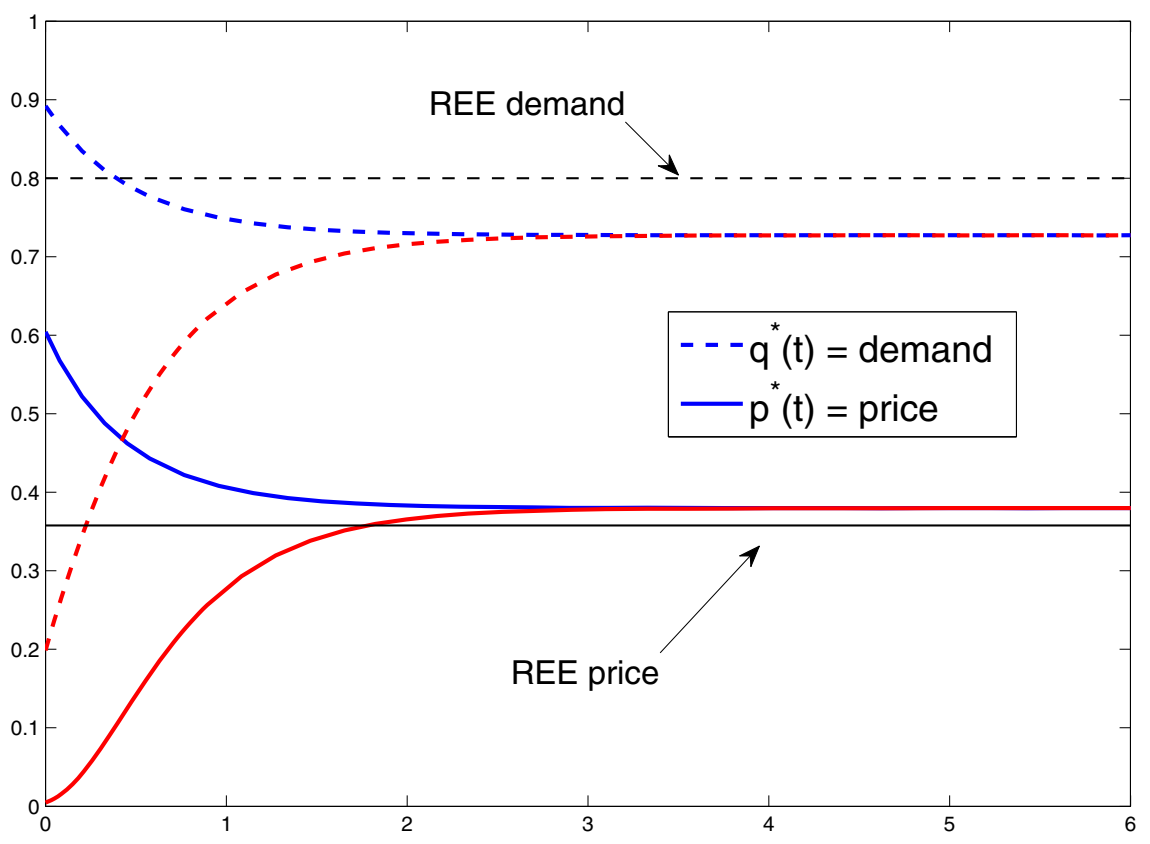

Fig. 1. Trajectories for the optimal pricing policy, $p(t)$, and the demand, $q(t)$, in the quadratic case, $F(a)=a^{2}$. specify a probability measure over the set of feasible controls at each time $t$. Therefore, at any time $t$, define a generalized control as a probability measure $\mu(\cdot, t)$ on $[0,1]$, and for each $\mu$ define

$$
\begin{aligned}
& \bar{a}(\mu)=\int a d \mu(a), \\
& \bar{F}(\mu)=\int F(a) d \mu(a) .
\end{aligned}
$$

Then the immediate return at time $t$ from $\mu(\cdot, t)$ and the law of motion, $q$, are given by

$$
\bar{a}(\mu[\cdot, t])[q(t)]^{2}, \quad q^{\prime}(t)=k[1-\bar{F}(\mu)-q] .
$$

One can show that there is an optimal generalized control that can be represented by a policy that maps each current state into a probability measure on the unit interval (5). In fact, this mapping takes a form that is analogous to the jump target policy in the uniform case.

Let the target be

$$
\sigma=\frac{2 k}{3 k+r}
$$

and define a generalized policy,

$$
\begin{gathered}
\mu_{\sigma}(1, q)=\left\{\begin{array}{cc}
0, & q<\sigma, \\
1-\sigma, & q=\sigma, \\
1, & q>\sigma,
\end{array}\right. \\
\mu_{\sigma}(0, q)=1-\mu_{\sigma}(1, q) .
\end{gathered}
$$

Theorem 5. If the $c d f F$ is strictly concave, then the generalized target policy given by [21] is optimal among all generalized policies.

Note that the optimal generalized policy is the same for all cdfs $F$ in the class covered by Theorem 5 . The interpretation of a generalized policy presents some difficulties. We conjecture that the supremum of the monopolist's profit can be approximated arbitrarily closely by ordinary policies that oscillate arbitrarily quickly, as in the example of "viscous demand," analyzed in ref. 5.

\section{Extensions of the Model of Myopic Consumers}

We now present two extensions of our model of myopic consumers. In each extension, we assume bounded attention and the uniform distribution of consumer types, but change the consumers' model of short-run demand expectation. Each extension establishes that the seller's optimal price trajectory retains the "jump target policy" structure derived in Theorem 4, although the level of the demand target and the steady-state price vary in the two cases.

An Evolving Consumer Population. In the first extension we assume that, roughly speaking, in each period of length $h$ a fraction $c h$ of adopters and nonadopters are randomly replaced by nonadopters. Then the process proceeds as in the myopic case. (See SI Text for a precise description.) As before, we denote the demand and price at time $t$ by $q(t)$ and $p(t)$, respectively, and let $a(t)=p(t) / q(t)$. For continuous time, the demand is given by the differential equation

$$
q^{\prime}(t)=k(1-\boldsymbol{a}(t))-(k+c) q(t), \quad 0 \leq q(t) \leq 1,0 \leq a(t) \leq 1 .
$$

For example, for any price trajectory that causes an increase in demand, the positive rate of consumer replacement slows down the rate at which demand increases, in comparison with the case in which $c=0$.

For any demand $q$, the control $a$ that makes the time derivative zero-the stay-where-you-are control-is

$$
A_{c}(q)=1-\left(1+\frac{c}{k}\right) q
$$

We define a jump target policy as before (Eq. 18):

Theorem 6. The seller's optimal price trajectory is generated by a jump target policy, with target and price at the target, resp.,

$$
\begin{gathered}
\sigma_{c}=\frac{2 k}{3(k+c)+r}, \\
P_{c}\left(\sigma_{c}\right)=A_{c}\left(\sigma_{c}\right) \sigma_{c}=\frac{2 k[(k+c+r)]}{[3(k+c)+r]^{2}} .
\end{gathered}
$$

Myopic and "Stubborn" Consumers. Finally, we discuss a model in which consumers are something between myopic and stubborn. 
Rather than basing their expectation of total demand in the next period on the current period's demand level, consumers who pay attention to the monopolist's price announcement partly base their prediction on a stubborn assessment, $\omega$, of the total demand for the good. The extent to which they base their expectation on $\omega$ is determined by a parameter $\gamma$, where $0 \leq \gamma \leq 1$. This gives

$$
q_{E}(t)=\gamma q(t)+(1-\gamma) \omega
$$

and the control variable is given by

$$
\boldsymbol{a}(t)=\frac{p(t)}{\gamma q(t)+(1-\gamma) \omega} .
$$

For a given $\gamma$ and $\omega$, let $\pi^{*}$ be the optimal target policy, and denote its target as $\sigma(\gamma, \omega)$. Theorem 7 confirms that the structure of the pricing policy prescribed in the previous section is qualitatively robust to this extension. However, the demand target is always lower.

Theorem 7. With the expected demand function given by Eq. 25:

i) The monopolist's optimal price trajectory is generated by the jump target policy with target $\sigma(\gamma, \omega)$;

ii) $\sigma(\gamma, \omega)$ is strictly increasing in $\gamma$ and has the following values at its end points,

$$
\begin{aligned}
& \sigma(0, \omega)=\frac{k}{2 k+r}, \\
& \sigma(1, \omega)=\frac{2 k}{3 k+r}
\end{aligned}
$$

iii) $\sigma(\gamma, \omega)$ is strictly decreasing in $\omega$.

Note that the demand target of Theorem 4 is a limiting case of the demand target above, when $\gamma=1$. $i i$ and iii have a simple intuitive explanation. An increase in the installed base for a network good benefits the seller in two ways: through the direct increase in demand and by increasing the willingness to pay of consumers. It is the latter property that increases the monopoly demand for the good beyond what a normal good would enjoy. Therefore, at any given stubborn expectation $\omega$, a decrease in the weight $\gamma$ placed on the current demand makes the good seem "less like" a network good and more like a normal good with an exogenously specified value that is proportionate to $\omega$, thus reducing the steady-state user base that the seller finds optimal. Correspondingly, for any given $\gamma$, an increase in $\omega$ reduces the fraction of perceived user value that is influenced by actual current demand and increases the corresponding fraction influenced by the stubborn expectation. One might therefore expect

1. Gabel D (1994) Competition in a network industry: The telephone industry, 1894-1910 J Econ Hist 54(3):543-572.

2. Temin P, Galambos L (1987) The Fall of the Bell System (Cambridge Univ Press, Cambridge, MA).

3. Mendelson H (2002) "PayPal," Case Number EC-27 (Graduate School of Business, Stanford University, Stanford, CA).

4. Radner R (2003) Viscous demand. J Econ Theory 112(2):189-231.

5. Radner R, Richardson T (2003) Monopolists and viscous demand. Games Econ Behav 45:442-454.

6. Weber T (2006) An infinite horizon maximum principle with bounds on the adjoint variable. J Econ Dyn Control 30:229-241.

7. Sundararajan A (2007) Local network effects and complex network structure. $B E J$ Theor Econ 7(1), 10.2202/1935-1704.1319. outcomes that are qualitatively similar to those of the base model if, rather than being a pure network good as we have assumed, a fraction of the willingness to pay for the good is independent of the demand $q$.

\section{Future Research Directions}

We have noted that most markets for network goods are not "pure monopolies" or "purely competitive." The analysis of oligopolies with unboundedly rational firms but boundedly rational consumers would naturally use the theory of dynamic strategic games. One of us has studied a duopoly model from this point of view, with consumers with bounded attention, but with no network effects (4). The results have the same flavor as those of the present paper, but there is a continuum of equilibria. The extension to models with network effects would appear to pose significant analytical challenges.

Finally, as in some recent models of local network effects (e.g., ref. 7), the rate at which consumers pay attention to products may not be constant across the population, but may be influenced by the adoption decisions of other consumers to whom one is locally "connected." This represents an interesting extension to our model of bounded rationality, one that is especially pertinent to network goods, and a direction of research we hope to pursue in the future.

\section{Bibliographic Notes and References}

The static model of network effects underlying our dynamic model is based on ref. 8 , which provided the first model of monopoly pricing for a network good, and on ref. 9. Early analyses of oligopolistic markets with network effects appeared in refs. 10 and 11. These and most subsequent analyses used models of consumer demand based on rational expectations. A notable exception is ref. 12, which can be interpreted as a model of "diffusion." In particular, a special case of their model leads to a law of motion that is mathematically isomorphic to our benchmark case of purely myopic consumers with a uniform distribution of types, and for that case they derive the optimal price trajectory. Another exception is ref. 13, which analyzes a nonstrategic duopoly with prices that are fixed exogenously and consumers whose demand is sequentially adjusted myopically and shows that, asymptotically, one of the firms will capture the entire market, but cannot predicit ex ante which firm will "win."

A complete review of the literature on network goods is beyond the scope of this paper, but some further notes are provided in SI Text.

ACKNOWLEDGMENTS. The authors thank Philipp Afeche, Kenneth Arrow Yannis Bakos, Toker Doganoglu, Nicholas Economides, Joseph Harrington, Urszula Ledzewicz, and Timothy Van Zandt and workshop participants at Boston University, INSEAD, New York University, Stanford University, the University of Minnesota, the Workshop on Information Systems and Economics, the National Bureau of Economic Research/National Science Foundation (NSF) Decentralization Conference, the North American Winter Meeting of the Econometric Society, and the Kiel-Munich Workshop on the Economics of Network Industries. A.R. was partially supported by NSF Grant DMS-1016136.

8. Rohlfs J (1974) A theory of interdependent demand for a communication service. Bell J Econ 5(1):16-37.

9. Economides N (1996) The economics of networks. Int J Ind Organ 14:673-699.

10. Katz M, Shapiro C (1985) Network externalities, competition and contracting. Am Econ Rev 75:424-440.

11. Farrell J, Saloner G (1985) Standardization, compatibility, and innovation. Rand J Econ 16(1):70-83.

12. Dhebar A, Oren S (1985) Optimal dynamic pricing for expanding networks. Mark Sci 4: 336-351.

13. Arthur B (1989) Competing technologies, increasing returns, and lock-in by historical events. Econ J 99(394):116-131. 